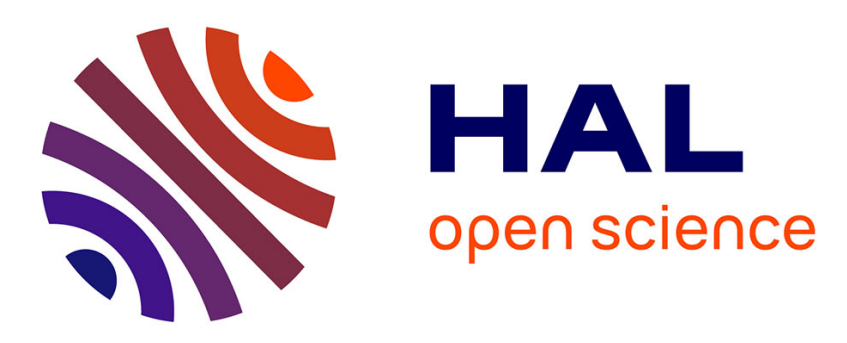

\title{
FEM technique for modeling eddy-current testing of ferromagnetic media with small skin depth
}

\author{
H. Zaidi, Laurent Santandrea, Guillaume Krebs, Yann Le Bihan, E.
}

Demaldent

\section{- To cite this version:}

H. Zaidi, Laurent Santandrea, Guillaume Krebs, Yann Le Bihan, E. Demaldent. FEM technique for modeling eddy-current testing of ferromagnetic media with small skin depth. IEEE Transactions on Magnetics, 2014, 50 (2), pp.7003004. 10.1109/TMAG.2013.2283246 . cea-01845468

\section{HAL Id: cea-01845468 https://hal-cea.archives-ouvertes.fr/cea-01845468}

Submitted on 9 Jan 2019

HAL is a multi-disciplinary open access archive for the deposit and dissemination of scientific research documents, whether they are published or not. The documents may come from teaching and research institutions in France or abroad, or from public or private research centers.
L'archive ouverte pluridisciplinaire HAL, est destinée au dépôt et à la diffusion de documents scientifiques de niveau recherche, publiés ou non, émanant des établissements d'enseignement et de recherche français ou étrangers, des laboratoires publics ou privés. 


\title{
FEM Technique for Modeling Eddy-Current Testing of Ferromagnetic Media With Small Skin Depth
}

\author{
Houda Zaidi ${ }^{1,2}$, Laurent Santandrea ${ }^{1}$, Guillaume Krebs ${ }^{1}$, Yann Le Bihan ${ }^{1}$, and Edouard Demaldent ${ }^{2}$ \\ ${ }^{1}$ Laboratoire de Génie Electrique de Paris, UMR 8507 CNRS, SUPELEC, University of Paris-Sud \\ and UPMC LGEP, Gif-sur-Yvette 91192, France \\ ${ }^{2}$ Laboratoire d'Intégration des Systèmes et des Technologies, CEA, LIST, Gif-sur-Yvette 91191, France
}

\begin{abstract}
The modeling of eddy-current testing problems, by means of finit elements, requires us to carefully consider the skin effect. When the material to inspect has a small skin depth compared with the other dimensions of the problem, which is a typical situation in the case of ferromagnetic media, the classical FEMs can become unsuitable in terms of mesh quality or computation time. In this paper, the overlapping FEM is implemented to effi iently consider the skin effect. 3-D problems are studied and the validity of the proposed approach is shown.
\end{abstract}

Index Terms — Eddy currents, f nite element analysis, magnetic materials, magnetic multilayers, non-destructive testing.

\section{INTRODUCTION}

$\mathbf{T}$ HE non-destructive testing by means of the ECT is nowadays frequently used to evaluate the properties of conducting materials. The interpretation of the signal variations received by the ECT probe (due for instance to a crack) involves the use of mathematical models. These models permit also to study conf gurations that are diff cult to reproduce experimentally.

The FEM is often chosen due to its high adaptability when using simplex elements [1] (triangles in 2-D or tetrahedra in 3-D). However, a correct modeling of ECT requires some rules: 1) accurate meshes for the probe and the surrounding air; 2) fi e mesh in the liftoff (distance between the probe and the conducting part); and 3) usually (considering frstorder elements) at least three layers of f nite elements have to be used in the skin depth of the inspected conductor. This last condition becomes critical when considering ECT of ferromagnetic materials having a low skin depth. Indeed the meshing of these materials will result either in highly distorted simplex fi ite elements or in a high density mesh leading to a huge number of degrees of freedom (DOF). This paper proposes an eff cient technique for modeling ECT of ferromagnetic materials based on the overlapping method. In the previous works, numerical techniques based on this method have been proposed to consider a thin geometrical domain thanks to a layer of overlapping elements [2]-[5]. In this paper, we propose to adapt this technique to model eff ciently the skin effect in low skin depth domains using several layers of overlapping elements. The overlapping method is used to obtain a prismatic mesh in the frst skin depths of the ferromagnetic material and to connect this mesh to the initial tetrahedral mesh of the remaining of the study domain.

The frst part of this paper describes brief $y$ the used f nite element formulations. In the second part, the overlapping FEM is presented. Then, some explanations are given about the proposed approach to consider the skin effect. Finally, two test cases are considered and results obtained with the implemented method are compared with analytical and experimental data. The frst test case includes a ferromagnetic piece free from defect, while the second one is dedicated to model a fl wed ferromagnetic piece.

\section{Finite Element Formulations}

There are two families of formulations for solving Maxwell's equations. One is based on the calculation of the electric feld $\boldsymbol{e}$ and the other on the calculation of the magnetic fiel $\boldsymbol{h}$ [6]. In either electric or magnetic formulation, potentials are generally used to describe the feld. In the magnetic formulation, the magnetic fiel $\boldsymbol{h}$ is described as a function of the electric vector potentials $\boldsymbol{t}$ and $\boldsymbol{t}_{\mathbf{0}}$ and with the help of the magnetic scalar potential $\varphi$

$$
h=t+t_{0}-\operatorname{grad} \varphi .
$$

The electric current vector potentials $\boldsymbol{t}$ and $\boldsymbol{t}_{\mathbf{0}}$ come from the relations curl $\boldsymbol{t}=\boldsymbol{j}$ and curl $\boldsymbol{t}_{\mathbf{0}}=\boldsymbol{j}_{\mathbf{0}}$, where $\boldsymbol{j}$ is the density of eddy currents and $\boldsymbol{j}_{\mathbf{0}}$ is the density of source current. In the electric formulation, with the introduction of a magnetic vector potential $\boldsymbol{a}$ and an electric scalar potential $\psi$, the electrical fiel $\boldsymbol{e}$ is expressed as

$$
\boldsymbol{e}=-\partial_{t}(\boldsymbol{a}+\operatorname{grad} \psi) .
$$

The potential $\boldsymbol{a}$ comes from the relation $\boldsymbol{b}=\operatorname{curl} \boldsymbol{a}$, where $\boldsymbol{b}$ is the magnetic $\mathrm{fl} \mathrm{x}$ density. The potentials are discretized by Whitney elements. In this paper, the 3-D geometry is discretized using tetrahedral. The modeling is made in harmonic regime. The obtained algebraic system is solved using a Jacobi preconditioned conjugate gradient method.

\section{OVERLAPPING FEM}

The overlapping FEM has been $\mathrm{frst}$ introduced in 2-D and applied recently in 3-D for ECT applications in [3] and [4]. Its principle is to connect two domains separated by an initially unmeshed region [see Fig. 1(a)]. The shape functions belonging to the nodes $i$ and $j$ are def ned only in $D_{1}$ and $D_{2}$, 


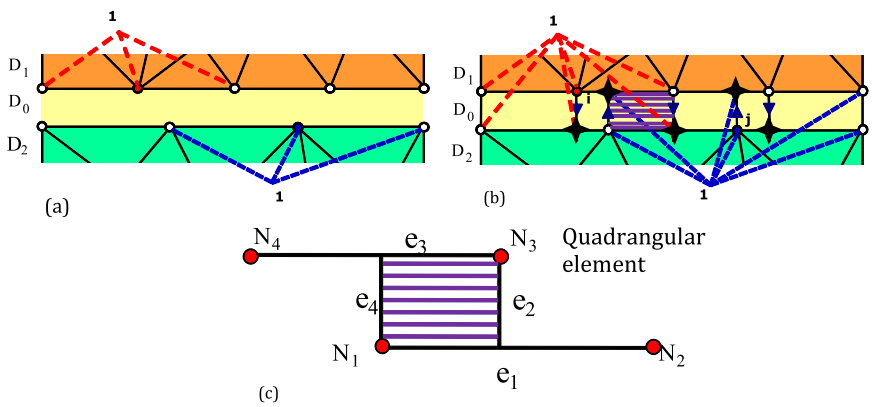

Fig. 1. (a) Two domains independently meshed. (b) Nodal projections. (c) Nodal and edge unknowns associated with a quadrilateral element.

respectively. A projection of the nodes of $D_{1}$ is performed in $D_{0}$ up to the boundary of $D_{2}$ and reciprocally from $D_{2}$ toward $D_{1}$ for the $D_{2}$ nodes, see Fig. 1(b).

This double projection creates overlapping elements. These elements are the support of shape functions, which permit to ensure the continuity of the interpolated values. The projected nodes [stars in Fig. 1(b)] are virtual and are not the support of DOF. They are just used to determine integration areas [hatched area in Fig. 1(b)], where the different integral terms of the FEM formulation are evaluated. New shape functions are introduced in $D_{0}$. These functions are represented in Fig. 1(b) by quadrilateral elements (or prisms in 3-D) that overlap in $D_{0}$. In this region, integral terms are computed in the area where shape functions are commonly non-zero [see the hatched area in Fig. 1(b) related to the nodes $i$ and $j]$ and added to the matrix system. Depending on the used formulation, new vertical edges have to be added in the problem.

For each quadrilateral element (or prismatic element in 3-D), nodal and edge unknowns are considered. The nodal unknowns are the four nodes of the two edges from which derives the quadrilateral element (or the six nodes of the two triangles from which derives the prism). For the hatched area in Fig. 1(c), the nodal unknowns are associated to $N_{1}$, $N_{2}, N_{3}$, and $N_{4}$. In a vector potential formulation, unknowns on vertical edges are added [5]. For the hatched area in Fig. 1(c), the edge unknowns are associated to the edges $e_{1}, e_{2}$, $e_{3}$, and $e_{4}$. It follows that the edge unknowns are composed of the two edges of the two lines that derives the quadrilateral element (or the six edges of the two triangles) in addition the two vertical edges of the quadrilateral (or the three vertical edges of the prism). This is shown, in the 3-D case, in Fig. 2.

\section{Modeling of the Skin Effect}

In the ECT of ferromagnetic materials, because of the high value of the magnetic permeability, the skin depth is usually small compared with other geometrical parameters. The eddycurrent density is important in the skin depth and decreases quickly and approximately exponentially. A f ne mesh is then required in the skin depth of the ferromagnetic material. However, since the skin depth is very small, it is diff cult to generate a specif $\mathrm{c}$ mesh that contains enough elements in skin depth without a very high total number of DOF in the mesh and, above all, without reducing the quality of the mesh. Indeed, the meshing of the skin depth with several layers of

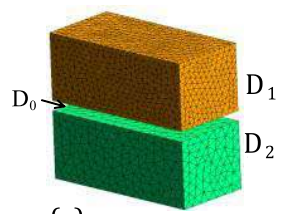

(a)

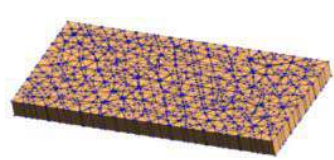

(c)

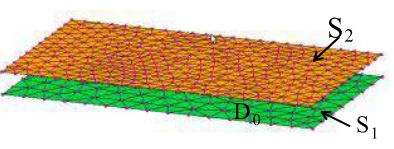

(b)

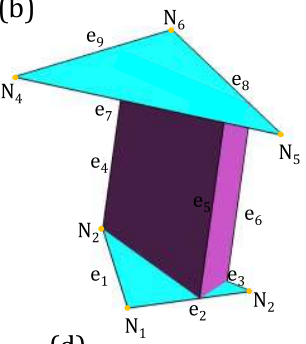

(d)
Fig. 2. (a) Two meshes separated by a free volume $D_{0}$. (b) Two surfaces that bound $D_{0}$. (c) Mesh of $D_{0}$. (d) Unknowns associated with an integration area (prism).

equilateral elements leads to a large number of unknowns. On the contrary, a less dense mesh can lead to highly distorted elements and sometimes to an insuff cient discretization to model induced currents.

The proposed approach considers the frst skin depths with multiple layers of overlapping elements. With this approach, it is possible to consider the high skin effect occurring in ferromagnetic materials without the previously presented mesh diff culties. Indeed, the principle of this method is to mesh the area with prismatic elements which, unlike the tetrahedral elements, can be flattene to a large extent without critically degrading their quality. It is possible to use overlapping element layers with a non-uniform thickness distribution to consider the feld evolution in the depth. For example, an exponential distribution according to the depth, while respecting the requirement to have at least three or four layers of elements in the skin depth, should allow to discretize f nely the material surface and more coarsely its depth with the purpose to limit the number of DOF. Fig. 3 shows the overlapping layer distribution adopted in this paper.

With the overlapping method, different triangular discretization can also be adopted from one layer to the other, notably in the case of materials of non-canonical shape. This justif es the choice of the overlapping method compared with other methods such as shell element method.

\section{TEST CASE AND COMPARISONS}

\section{A. Studied Configu ation}

In this paper, two test cases to validate the implementation of the overlapping approach for the ECT of ferromagnetic materials will be treated. The fir t problem concerns the ECT of a ferromagnetic medium free from defect. The aim of the second problem is to obtain the response of an ECT probe to a faw (slot) in a ferromagnetic material. Fig. 4(a) [respectively Fig. 4(b)] shows the conf guration of the frst test case (respectively second). The parameters of the two problems are given in Table I [7]. 


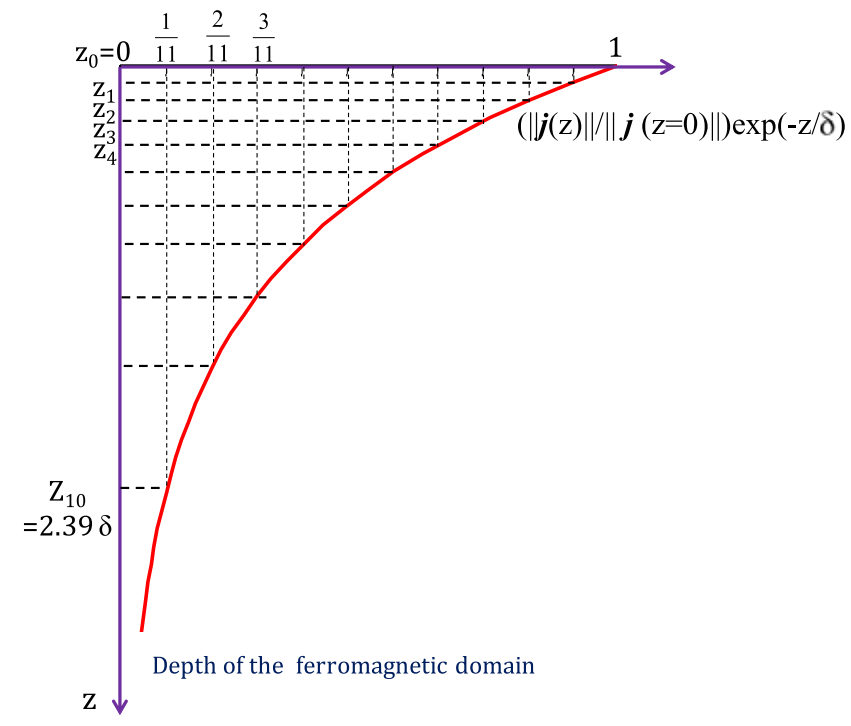

Fig. 3. Discretization of the fir $\mathrm{t}$ skin depths of the ferromagnetic domain in several layers of overlapping elements.

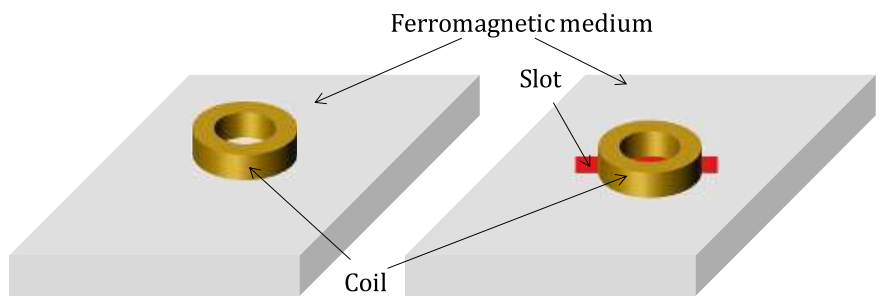

(a)

(b)

Fig. 4. Configur tions of the ECT test cases. (a) Defect-free ferromagnetic medium. (b) Flawed ferromagnetic medium.

TABLE I

Parameters of the Test Case [7]

\begin{tabular}{|l|c|}
\hline \multicolumn{2}{|c|}{ parameters of the coil } \\
\hline External radius of the coil & $10.7 \mathrm{~mm}$ \\
\hline Internal radius of the coil & $6.88 \mathrm{~mm}$ \\
\hline Height of the coil & $5 \mathrm{~mm}$ \\
\hline Number of turns of the coil & 410 \\
\hline Thickness of the lift-off & $1.9 \mathrm{~mm}$ \\
\hline \multicolumn{2}{|c|}{ Parameters of the plate } \\
\hline Thickness of the plate & $10 \mathrm{~mm}$ \\
\hline Conductivity of the plate & $3.47 \mathrm{MS} / \mathrm{m}$ \\
\hline Relative permeability of the plate & 85 \\
\hline \multicolumn{2}{|c|}{ Parameters of the slot (flaw) } \\
\hline Depth of slot & $6 \mathrm{~mm}$ \\
\hline Length of slot & $40 \mathrm{~mm}$ \\
\hline Width of slot & $0.43 \mathrm{~mm}$ \\
\hline
\end{tabular}

\section{B. Flaw-Free Material}

The studied problem is a simple coil above a ferromagnetic plate without defect. It comes from a conf guration proposed in [7] with associated experimental and analytical results. The main parameters of the problem are given in Table I (except for the faw parameters, which are irrelevant here).

The working frequency range is very large from $0.1 \mathrm{kHz}$ to $100 \mathrm{MHz}$. Consequently, the skin depth in the plate varies (a)

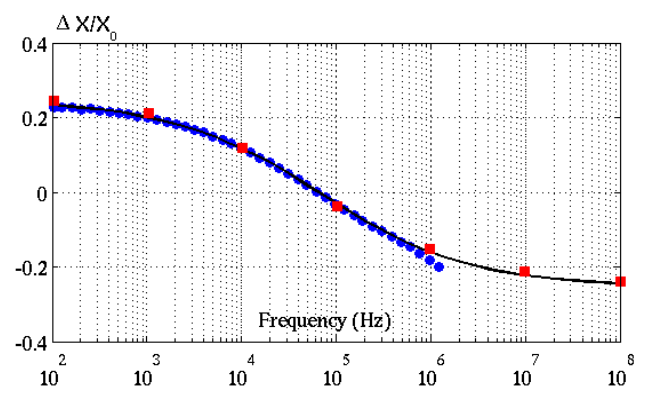

(b)

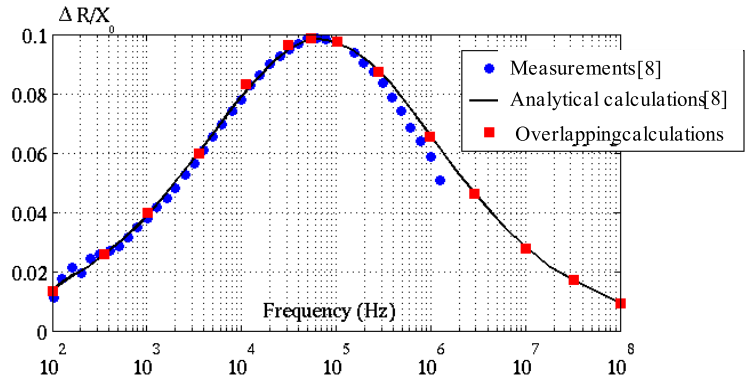

Fig. 5. (a) Normalized reactance and (b) resistance variation resulting from the presence of the plate as a function of the frequency.

considerably from $2.9 \mathrm{~mm}$ to $2.9 \mu \mathrm{m}$. It was chosen to work with 10 overlapping layers whose thicknesses are equal to $(1 \leq i \leq 10)$

$$
t_{i}=\left(z_{i}-z_{i-1}\right) \quad \text { with } z_{i}=\ln (11 /(11-i)) \delta
$$

where $\delta$ is the skin depth of the plate. By this way, the fi st $2.39 \delta$ of the plate depth are discretized with the overlapping method (Fig. 3). The remainder of the plate (inside) is meshed with tetrahedral elements. For the lowest frequencies $(\leq 10 \mathrm{kHz})$, the thicknesses of the overlapping layers have been kept constant to avoid elements of too large thickness. For these low frequencies, the classical FEM could be used. In the considered problem, the thickness of the liftoff (coil plate distance) is not very small compared with the other dimensions of the problem; hence, the liftoff domain is meshed with tetrahedral elements.

The proposed approach has been implemented and the impedance variation $\Delta Z=\Delta R+j \Delta X$ of the coil due to the presence of the plate is calculated. The evolution of $\Delta R$ and $\Delta X$ normalized by the reactance $X_{0}$ of the coil in air is shown in Fig. 5 over the frequency range. These results are compared with analytical and experimental data issued from [7].

The two formulations presented previously have properties of duality [6]. To obtain better results for both magnetic and electric felds, it is recommended to solve a given problem with both formulations and use averaged values.

It can be noticed that the results are in good agreement. The method allows extending very signif cantly the frequency range of application of the FEM by comparison with classical FEM. Limitations of the classical FEM with a meshing constituted only of tetrahedral elements (and of other numerical techniques) can be found in [8], where the same test case was considered. This reference shows that the classical FEM results begin to diverge for high frequency (above several kilohertz) due to the increasing skin effect. This demonstrates the interest of the approach developed in this paper. 


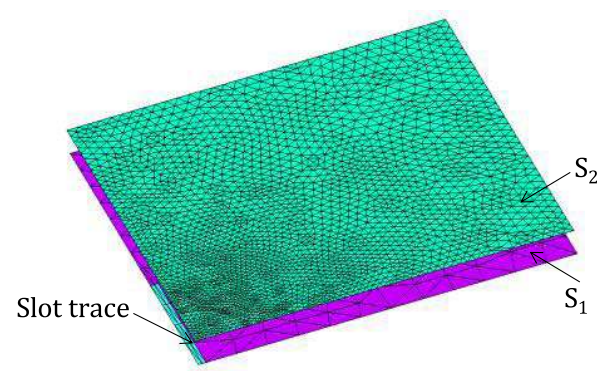

(a)

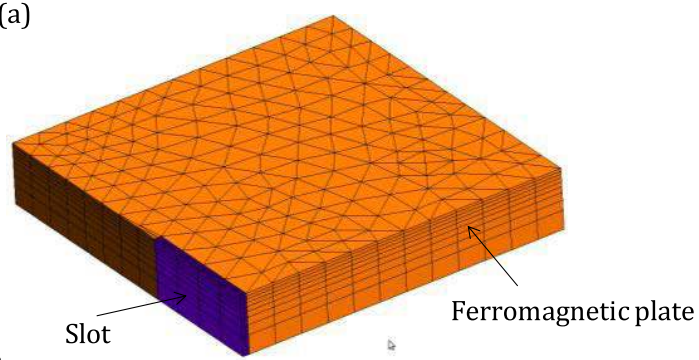

(b)

Fig. 6. (a) Traces of the tetrahedral meshes on the top and on the bottom of the overlapping domain. (b) Prismatic mesh of plate and the slot.

(a)

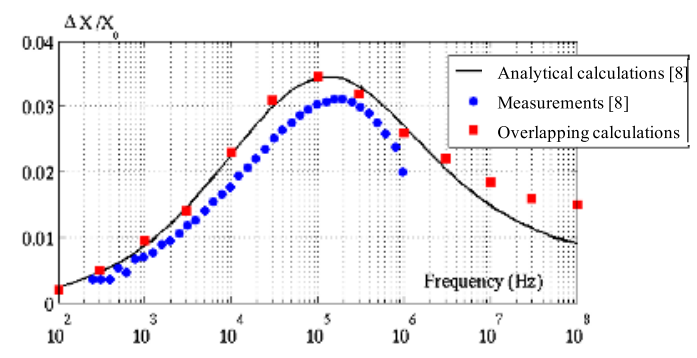

(b)

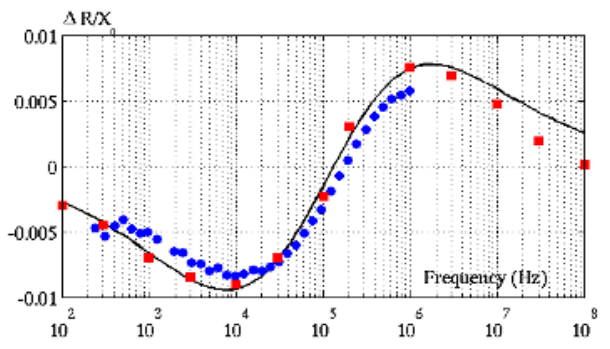

Fig. 7. (a) Normalized reactance and (b) resistance variation resulting from the presence of the slot in function of the frequency.

\section{Flawed Plate}

In this section, the overlapping method is applied to simulate the ECT signal induced by a f aw in a ferromagnetic plate. The probe is centered on the $\mathrm{fl} \mathrm{w}$ that is constituted by a long slot whose parameters are presented in Table I. The other parameters of this second problem are the same as the previous one.

The coil impedance variation resulting from the presence of the slot is calculated for the same wide range of frequency than previously (from $0.1 \mathrm{kHz}$ to $100 \mathrm{MHz}$ ).

For this example, the top part of the $\mathrm{faw}$ is also considered with overlapping elements. To do this, either $S_{1}$ or $S_{2}$ (boundary surfaces of the overlapping domain, Fig. 2) has to contain a 2-D mask of the slot [Fig. 6(a)]. The discretization of the two surface $S_{1}$ and $S_{2}$ can be different. As previously, the frst $2.39 \delta$ of the plate depth and of the slot is meshed with the overlapping method. The remaining depth of the plate and of the slot is meshed with tetrahedral elements.
The average value (calculated from the two formulations) of impedance variation $\Delta Z=\Delta R+j \Delta X$ of the coil due to the defect is calculated. Fig. 7 shows the evolution of $\Delta R$ and $\Delta X$ normalized by the reactance of the coil in air, in function of the frequency. These results are compared with analytical and experimental data issued from [7].

These results show a good agreement between modeling results and measurements of [7] and the results provided by the overlapping method. However, it can be noticed that the overlapping results begin to deviate from the analytical ones above several megahertz. From the overlapping method point of view, this discrepancy could be due to the triangular discretization of the overlapping domain in its horizontal plane, which limits the smallness of the f nite elements around the faw. Nevertheless, the applicability of the FEM for the modeling of $f$ aw detection in ferromagnetic media is considerably increased using the overlapping method.

\section{CONCLUSION}

In this paper, a new approach based on the overlapping method for modeling the ECT of thin skin depth media, such as ferromagnetic media, has been proposed. This approach has been applied into two test cases involving $\mathrm{f}$ aw free and $\mathrm{fl}$ wed ferromagnetic plate, respectively. It can be concluded from the tests that the overlapping method allows an eff cient modeling of the ECT of low skin depth media. This method can be also applied to consider non-homogeneous media such as stratif ed media. It allows meshing thin regions with adapted elements with a great fl xibility, thanks to the non-conformity of the mesh provided by the method. It can also be noticed that with this method the displacement of the probe can be considered without remeshing.

\section{ACKNOWLEDGMENT}

This work was supported by DIGITEO (Île-de-France Region) and CEA LIST.

\section{REFERENCES}

[1] R. Hamia, C. Cordier, S. Saez, and C. Dolabdjian, "Eddy-current nondestructive testing using an improved GMR magnetometer and a single wire as inducer: A FEM performance analysis," IEEE Magn. Soc., vol. 46, no. 10, pp. 3731-3737, Oct. 2010.

[2] H. Zaidi, L. Santandréa, G. Krebs, Y. Le Bihan, and E. Demaldent, "Eff cient FEM modelling of printed coils for eddy current testing," in Proc. Numelec Conf., Jul. 2012, pp. 1-4.

[3] H. Zaidi, L. Santandréa, G. Krebs, Y. Le Bihan, and E. Demaldent, "Modeling of thin conducting and magnetic layers in eddy current testing by overlapping f nite elements," Int. J. Appl. Electromagn. Mech., vol. 39, pp. 341-346, Sep. 2012.

[4] I. A. Tsukerman, "Overlapping finit element for problems with movement," IEEE Trans. Magn., vol. 28, no. 5, pp. 2247-2249, Sep. 1992.

[5] G. Krebs, T. Henneron, S. Clenet, and Y. Le Bihan, "Overlapping finit elements used to connect non-conforming meshes in $3 \mathrm{D}$ with a vector potential formulation," IEEE Trans. Magn., vol. 4, no. 5, pp. 341-346, May 2010.

[6] Z. Ren and A. Razek, "Computation of 3-D electromagnetic f eld using differential forms based elements and dual formulations," Int. J. Numer. Model., Electron. Netw., Devices Fields, vol. 9, nos. 1-2, pp. 81-98, Jan. 1996.

[7] R. J. Ditchburn, S. K. Burke, and M. Posada, "Eddy-current nondestructive inspection with thin spiral coils: Long cracks in steel," J. Nondestruct. Eval., vol. 22, no. 2, pp. 63-77, Jun. 2003.

[8] C. Zorni, C. Reboud, J. M. Decitre, L. Santandréa, Y. Le Bihan, S. Ventre, et al., "Modelling eddy current testing of ferromagnetic medium," Int. J. Appl. Electromagn. Mech., vol. 39, pp. 245-250, Sep. 2012. 